\title{
Le meurtrier et son double chez les Araweté : Un exemple de fusion rituelle
}

The murderer and his double among the Araweté: An example of ritual fusion (Brazil)

\section{Eduardo Viveiros de Castro}

Traducteur : Estela dos Santos Abreu

\section{(2) OpenEdition}

Journals

Édition électronique

URL : http://journals.openedition.org/span/1497

DOI : 10.4000/span. 1497

ISSN : 2268-1558

Éditeur

École pratique des hautes études. Sciences humaines

Édition imprimée

Date de publication : 1 août 1996

Pagination : 77-104

ISSN : 0294-7080

Référence électronique

Eduardo Viveiros de Castro, «Le meurtrier et son double chez les Araweté : Un exemple de fusion rituelle », Systèmes de pensée en Afrique noire [En ligne], 14 | 1996, mis en ligne le 17 avril 2014, consulté le 10 octobre 2020. URL : http://journals.openedition.org/span/1497 ; DOI : https://doi.org/ 10.4000/span. 1497 


\section{LE MEURTRIER ET SON DOUBLE \\ CHEZ LES ARAWETE : \\ Un exemple de fusion rituelle}

\section{par \\ Eduardo Viveiros de Castro}

Ce texte examine les relations entre le meurtrier et sa victime, telles qu'elles sont conçues et vécues chez les Araweté, une société de langue tupi-guarani habitant l'Amazonie orientale. Les matériaux ethnographiques ici présentés ont déjà fait l'objet d'autres publications ${ }^{1}$ où ils ont permis d'entamer une réflexion sur le rôle de l'altérité dans la détermination de la topique du socius amérindien. Nous reprenons maintenant ces faits dans une perspective légèrement plus spéculative, ayant pour objet la dynamique identitaire que présuppose le statut de tueur, personnage central de la symbolique de la guerre en Amazonie. Il s'agit donc moins de discuter les fonctions (politiques, idéologiques ou autres) associées à ce statut que de cerner les procédés rituels à l'œuvre dans sa constitution.

1 Viveiros de Castro, $1992 a, 1993 a, 1993 b$.

Destins de meurtriers

Systèmes de pensée en Afrique noire, 14, 1996 


\section{Eléments de cosmologie}

L'univers des Araweté, une petite société tupi-guarani habitant le Moyen Xingu, au Brésil, a pour origine et fondement la distinction entre l'humanité (Bide) et la divinité (Mal). Cette distinction est contemporaine de la séparation qui a eu lieu entre ciel et terre, au début des temps : suite à une querelle entre ceux qui deviendront des dieux et ceux qui resteront des hommes, les premiers partent tout en soulevant le firmament et emportant avec eux la science de l'éternelle jeunesse et de l'abondance sans travail. Depuis lors, les humains se nomment les « abandonnés » (hena mi re), ceux qui ont été délaissés par les Mal. Tout ce qui existe sur la terre est pensé comme partageant, face aux êtres et aux choses qui ont accédé à l'échelon céleste, une même condition d'abandon et de minorité ontologique : taille chétive, vulnérabilité aux maladies, mortalité.

Pourtant, parmi tous les êtres périssables de notre palier cosmique, les humains occupent une place à part : ils sont « ceux qui iront » (uha me'e rin), les seuls qui, après la mort, iront se joindre aux Mai. Au moment de la mort, une partie de la persona humaine, l'âme ou principe conscient (in), monte aux cieux et y est reçue par les Mat-hete, les " vrais dieux ", la race divine plus directement en rapport avec l'himanite ${ }^{2}$. Les Mal-hete ressemblent aux Araweté, tout en étant plus beaux, plus grands et plus forts que les humains, ce qui est le cas pour tout ce qui est de l'ordre céleste. La présentation corporelle des dieux est hyperbolique par rapport à celle des humains (parure de plumes d'ara rouges, boucles d'oreilles au duvet jaune de toucan et bleu de cotinga) ; mais ils exhibent aussi de fins dessins géométriques sur leurs corps, faits avec du jus noir de yanipa (Jenipa americana). Ce style de décoration est caractéristique des ennemis des Araweté, surtout les Kayapó. Les Araweté barbouillent leurs visages et leurs corps avec le jus de ce fruit, associé symboliquement au jaguar, quand ils partent en guerre ou vont à la chasse, mais ils ne s'en servent jamais pour décorer leurs corps.

2 Il y a des dizaines d'espèces divines portant des noms et attributs spécifiques. Le suffixe -hete indique l'exemplarité, l'authenticité ou l' * ipséité * du référent du concept qu'il vient modifier, servant encore comme marqueur d'emphase. 
Les $M a r$ se montrent donc sous une apparence qui mêle des signes propres aux Araweté et propres aux ennemis.

En effet, les Araweté affirment que les $\mathrm{Mal}$, bien qu'étant « comme nous », sont à la fois " comme des ennemis », non seulement parce qu'ils portent les mêmes peintures que les ennemis mais surtout car ils sont féroces et dangereux. Les Mal sont anthropophages : les âmes des morts à leur arrivée aux cieux sont tuées et mangées par eux. Ensuite ils les refont, plongeant les os de leurs victimes dans un bassin en pierre rempli d'une eau magique qui bout sans feu ; les morts alors ressuscitent et deviennent " comme les Mal" ", c'est-à-dire jeunes et beaux pour toujours. Les morts devenus divins se marient avec les dieux et, tous ensemble, retournent sur la terre pour partager à l'occasion des rituels la nourriture offerte par les humains au peuple céleste. Les chamans (peye) font des voyages aux cieux où ils s'entretiennent avec les dieux et les morts, les ramenant souvent sur la terre pour ces banquets rituels, ou tout simplement pour bavarder avec les vivants. Le chamanisme araweté est avant tout un moyen de maintenir les échanges entre les vivants et les Mal. Les humains offrent à manger aux dieux dans les deux sens, littéral et sexuel et, en retour, reçoivent des chants (la * musique des dieux » chantée par les chamans) ainsi que d'autres biens spirituels: la vie posthume aux cieux, bien entendu, mais aussi la continuité du monde, puisque la consommation cannibale et sexuelle des morts empêche les Mal de faire tomber le firmament en écrasant la terre.

Les dieux sont ambigus à plus d'un titre. A la différence de ce qu'on relève dans la plupart des groupes tupi-guarani, la cosmologie des Araweté ne conçoit pas les Mal comme des héros culturels, pères fondateurs ou maîtres de l'humanité ${ }^{3}$. Les dieux araweté sont à la fois au-delà et en-deçà de la culture : ils disposent d'une science chamanique absolue, capable de ressusciter les morts et de faire en sorte que les instruments travaillent tout seuls ; mais cela n'empêche pas qu'on les tienne pour des 'primitifs' (uka-hete me'e, " simplement existants " ou

\footnotetext{
${ }^{3}$ Le mot Maï est le correspondant araweté de Maira, nom très répandu chez les Tupi pour désigner les démiurges ou héros culturels qui se sont éloignés des hommes au début des temps.
} 
" sans culture ", comme on dit des animaux), comme des gens qui ne connaissent pas le feu ni les plantes cultivées. Ces conquêtes de la civilisation ne sont pas dues aux Mat; bien au contraire, c'est un humain qui les leur a apprises. Ainsi, bien qu'ils utilisent aujourd'hui une technologie culinaire semblable à celle des humains, un curieux épithète 'dionysiaque' continue de désigner les dieux comme sauvages : me'e wi a-re, «mangeurs de cru ", expression qui décrit de façon exemplaire les jaguars.

Il faut bien saisir ce que les Araweté veulent dire lorsqu'ils affirment que les Mal sont " comme des ennemis " (awin herin). Les dieux sont comme des ennemis car ils se conduisent vis-à-vis des morts araweté comme si ceux-ci étaient des ennemis : ils les tuent et les dévorent. Mais, s'ils agissent ainsi c'est que les morts se conduisent comme des ennemis face aux dieux : quelqu'un qui vient de mourir est un être laid, sale, bête et méchant, plein de rancune du fait d'être mort. A leur arrivée aux cieux, les âmes masculines sont accueillies par les dieux qui les pressent de demandes de cadeaux précieux ; aux âmes féminines, ils demandent des faveurs sexuelles. Très avares, les morts rechignent toujours aux avances des $\mathrm{Mal}$, se faisant tuer sur le coup. Les dieux se conduisent alors comme des ennemis ; mais, au fait, les vrais ennemis sont les morts, puisque les maîtres du point de vue céleste sont les dieux ; légitimes détenteurs de la position de sujet dans leur monde, il sont donc effectivement " comme nous" (bide herin) ${ }^{4}$.

\footnotetext{
${ }^{4}$ Le mot bïde, que j'ai traduit par * hurnanité *, veut dire aussi * nous *, * les gens * et " les Araweté *. Il ne s'agit pas d'un ethnonyme équivalent à "Araweté * (mot inventé par les Blancs) ou d'une 'autodésignation' substantive et distinctive - il faut rappeler que bïle est sous l'aspect syntaxique et sémantique l'équivalent de ñane, pronom de la première personne pluriel inclusif (opposé à ure, pluriel exclusif) -, mais bien d'une marque de position énonciative, c'est-à-dire qu'il s'agit en fait d'un 'pronom' qui marque la position de sujet, et non pas d'un 'nom propre'. Comme plusieurs sociétés amazoniennes (voir le cas exemplaire des Nambikwara : Price, 1987), les Araweté n'objectivent pas le sujet collectif par le moyen de noms de type ethnonymique, les réservant pour les autres, e'est-à-dire les ennemis (awin). Je pense que le célèbre 'ethnocentrismeprimitif' sous-jacent aux présumées autodésignationsethniques traduites comme * êtres humains *, * les vraies gens * etc. n'est qu'une illusion substantiviste. Voir, à cet égard, l'analyse de Vilaça (1992: 49-51) sur le pseudo-ethnonyme wari des Pakaa-Nova.
} 
L'ambiguïté ontologique des dieux reflete leur statut sociologique : futurs conjoints des morts, les Mal sont des affins des vivants. Les Mat-hete, en particulier, reçoivent l'epithete de ure tiwa oho, "nos redoutables affins potentiels ». Tiwa, affin potentiel ou cousin croisé, est une désignation qu'on applique à celui qui n'est pas parent ; chargée de connotations agressives, elle n'est jamais employée lorsqu'on s'adresse à un concitoyen. Un tiwă est presque le même qu'un awin, un ennemi, mais un ennemi pensé sous le mode d'une alliance possible. Les Araweté appellent tiwã n'importe quel non-Araweté avec qui ils établissent des rapports différents de ceux, généraux et impersonnels, qu'ils entretiennent avec les tribus ennemies en tant que collectivités.

Sauvages mais splendides, dangereux mais convoités par les humains, " omophages " mais disposant d'une surculture chamanique, cannibales mais alliés potentiels, les Mar sont marqués d'une ambivalence fondamentale. Ils sont à la fois l'idéal du Moi araweté et l'archétype de l'Autre. Les Araweté se regardent avec les yeux des dieux, mais en même temps ils ne peuvent regarder ceux-ci que de leur propre point de vue trop humain, c'est-à-dire terrestre et mortel.

\section{Le meurtrier et sa victime}

Si les morts araweté sont des ennemis soit face aux humains, car la mort engendre un fantôme terrestre qui hante les vivants jusqu'a la décomposition des chairs du cadavre, soit face aux dieux, car les âmes célestes se conduisent avec peu de civilité à leur arrivée au paradis, et si les Mal sont, à leur façon, également des ennemis, il n'y a pas de place aux cieux pour les vrais ennemis des Araweté. Les âmes des ennemis morts ne trouvent pas d'accueil, même cannibale, chez les Mar, qui les rejettent sur la terre où elles périssent pour de bon.

Mais quand il s'agit d'un ennemi tué par les Araweté, c'est tout autre chose. L'âme d'un moropl'nã (littéralement, meurtrier) araweté et celle de l'ennemi qu'il a tué montent ensemble aux cieux et y jouissent d'une situation privilégiée : elles fusionnent dans une entité duelle qui, comme nous le verrons, reçoit des Mal les égards et l'attention dus aux semblables. 
Après avoir tué (ou simplement blessé) un ennemi dans un accrochage, un homme "meurt " (umanun). Dès qu'il retourne au village, il tombe dans une sorte de stupeur, restant immobile pendant plusieurs journées, sans rien manger. Son corps est rempli du sang de l'ennemi, qu'il vomit sans arrêt. Cette mort n'est pas le résultat d'un simple éloignement de l'âme (qui peut se produire plusieurs fois au cours de la vie d'une personne), mais un vrai devenir-cadavre : le tueur entend le frôlement d'ailes des vautours qui se rassemblent autour de * son * corps mort, c'est-à-dire du corps de son ennemi abandonné dans la forêt ; il se perçoit «comme en train de pourrir », ses os se ramollissent, il pue.

Quand l'ennemi a été effectivement tué (et pas seulement blessé), l'état de mort du meurtrier dure environ cinq jours. Celui-ci doit ingérer une infusion amère d'écorce de iwirara'i (Aspidosperma sp.), identique a celle que boivent les femmes pendant leurs règles et les parents en couvade. Il ne peut absolument pas toucher la dépouille de sa victime, sous peine de voir son propre ventre gonfler et éclater, dans une sorte d'accouchement mortel.

En outre, le tueur est soumis à un interdit plus long : pendant plusieurs semaines après son exploit, il ne peut pas avoir de relations avec sa femme. L'esprit de l'ennemi est « sur lui », et serait le premier à la pénétrer sexuellement ; le meurtrier, "venant après l'ennemi ", serait mortellement contaminé par le sperme étranger.

La période d'abstinence se termine quand l'esprit de la victime décide d'aller au bout de la terre " chercher des chants". A son retour, il transmet ces chants au tueur pendant son sommeil, ainsi qu'une série d'anthroponymes qui seront donnés aux nouveaux-nés. Une nuit, l'esprit de l'ennemi réveille à l'improviste son tueur en l'exhortant : « allons, tiwã, lève-toi et dansons ! » L'esprit de l'ennemi est censé être vexé à l'égard de son tueur, mais en même temps lui est indissolublement lié. A la longue, cette rancune se change en amitié, la victime et son meurtrier devenant "comme des apihi-piha». Apihi-piha désigne la relation sociale la plus prestigieuse chez les Araweté : il s'agit d'une forme d'amitié rituelle où deux couples se partagent sexuellement leurs conjoints, faisant ensemble de longues expéditions de chasse en forêt 
et étant partenaires de choix dans les danses collectives à l'occasion des fêtes de boisson.

On peut donc remarquer un net progrès dans les rapports établis entre la victime et son tueur, allant de l'altérité meurtrière à l'identité fusionnelle : celui qui était un vrai ennemi, un awin, se transforme tout d'abord en tiwa, affïn potentiel ; par la suite, il devient ami rituel, une sorte de double social et affectif du moi, qui est au fond un anti-affin, car il s'agit de quelqu'un avec qui on partage son épouse au lieu de lui prendre ou de lui donner une sæur'; et, enfin, quand le tueur meurt, la victime (restant pour toujours « avec " [-rehewe] ou «dans " [-re] le tueur) lui devient consubstantielle, comme un appendice qui lui sert de trait distinctif par rapport au commun des mortels dans le monde céleste.

\section{La mort ventriloque}

Pendant la danse qui clôt la réclusion du meurtrier et célèbre la mort de son ennemi, l'esprit de celui-ci est censé se tenir derrière le meurtrier, qui est le chantre de la cérémonie. L'ennemi est son " professeur de chant » (marakã memo'o-hã), lui soufflant les paroles de la

\footnotetext{
${ }^{5}$ Au début, les relations sexuelles du tueur avec sa femme étaient dangereuses parce que le sperme de l'ennemi aurait pu le contaminer; par contre, la transformation ultérieure de celui-ci en apihi-pihã du tueur suggère précisément un mélange de spermes, du fait d'avoir partagé les mêmes femmes. Si cet accès commun aux épouses respectives sert, d'un côté, à distinguer les amis rituels des beaux-frères, de l'autre, il semble les assimiler à des germains de même sexe, lesquels jouent officieusement de cette prérogative. Pourtant, si les relations d'apihi-pihã peuvent être établies entre personnes reliées par une variété de liens de parenté préalables, elles excluent deux genres de lien : celui entre beaux-frères et celui entre germains. Deux frères réels ne peuvent jamais entrer en rapport d'amitié rituelle ; l'inversion de l'affinité ne reconduit donc pas à la simple consanguinité, mais crée une troisième position. De façon générale, les apihi-pihã sont recrutés dans la périphérie de la parentèle, c'est-à-dire dans la sphère où se trouvent les tiwã, non-parents ou parents éloignés qui peuvent être alternativement affinisés ou transformés en amis rituels.
} 
chanson qu'il doit prononcer et qui seront reprises par la communauté masculine du village, réunie autour du meurtrier ${ }^{6}$.

Les ennemis reçoivent en général deux épithètes assez révélateurs : $k a$ 'n nahi, " assaisonnement du caouïn " (la bière de maïs servie durant la danse rituelle), et maraka nin, " futur chant-musique ». Le premier est une claire allusion cannibale : si les Araweté ne mangent pas leurs ennemis, le cannibalisme étant réservé aux dieux, du moins s'en serventils pour " agrémenter " la boisson. Le second évoque le rôle principal des ennemis : apporter des paroles d'ailleurs.

Les danses araweté, surtout lors des fêtes de caouĩn (qui ont lieu plusieurs fois par an), se font toujours au son des awin maraka, des " chants-musique des ennemis ", expression à valeur aussi bien génitive que possessive. Ce genre musical présente une structure simple : quatre à huit vers répétés des dizaines de fois, selon un rythme binaire et une ligne mélodique assez monotone. Ils sont chantés à l'unisson, dans un registre grave, par toute la communauté masculine, après que le tueur en ait énoncé les paroles. Chaque ennemi mort peut procurer plusieurs chants à son tueur. Une fois prononcées au moment de la danse qui célèbre la fin de la réclusion du meurtrier, ces chansons d'ennemi tombent dans le domaine public, pouvant désormais être reprises par tout homme qui jouera le rôle de chantre d'une cérémonie de caouïn.

La complexité essentielle des chansons d'ennemi est due à leur étrange régime d'énonciation, hanté par le point de vue de l'ennemi. Le genre 'discours rapporte' propre aux récits traditionnels araweté atteint son plus grand rendement dans les awin marakan, creant un vertigineux jeu de miroirs entre les deux 'chanteurs', la victime et son meurtrier. Le sujet de l'enonciation est toujours la victime, qui peut parler en son propre nom mais qui peut aussi citer le discours d'un tiers.

6 Il faut observer que, si au début l'ennemi *venait devant * le tueur pendant la réclusion rendant dangereuses les relations sexuelles, pendant la danse guerrière il occupe une position derrière le tueur ; si auparavant il y avait le risque d'un mélange létal de spermes et une sorte de compétition entre ennemi et meurtrier, pendant la danse on voil s'établir une collaboration entre eux, manifestée par la répétition des mêmes paroles. 
Voyons, à titre d'exemple, une chanson attribuée à Yakati-ro, que lui avait apprise une victime de la tribu Parakanã en 1975 :

1. " "Je me meurs"

2. ainsi disait feu Moiwito ;

3. ainsi parlait ma proie,

4. ainsi parlait feu Koiarawï ;

5. "Dans sa large cour,

6. "Eeh!" a dit le Towaho,

7. "Voilà mon prisonnier,

8. dans la cour du grand oiseau." "

Le mort qui se dit en train de mourir, feu Moiwito ou Koiarawi, est un Araweté tombé sous les flèches parakanã peu avant l'expédition de représailles où l'ennemi qui parle dans ce chant a été tué par Yakati-ro. Ainsi, au vers $n^{\circ} 3$, l'ennemi-chanteur s'identifie comme étant le tueur de Moiwito, et cite les mots de sa victime: "Je me meurs ". Dans la deuxième partie du chant (marquée par une nouvelle cadence), le sujet de l'énoncé change. Le vers $n^{\circ} 5$ fait référence au vautour, signalé par l'euphémisme " grand oiseau " au vers $n^{\circ} 8$. La " cour du grand oiseau " est une métaphore macabre qui évoque la clairière ouverte par les vautours dans la forêt, là où gît le corps de l'ennemi tué, c'est-à-dire le cadavre du Parakanã, sujet de l'énonciation de ce chant, et non pas celui de Moiwito. Les paroles de cette seconde partie du chant sont attribuées au Towaho mentionné par l'ennemi au vers $n^{\circ}$ 6. Towaho est le nom d'une ancienne tribu ennemie des Araweté ${ }^{7}$ qui sert de synecdoque à " ennemi " dans de nombreux récits traditionnels. Mais dans ce chant le «Towaho»n'est autre que Yakati-ro lui-même, c'est-à-dire le tueur araweté qui est en train de chanter les mots que l'ennemi lui a appris. Du point de vue de la victime parakanã, son meurtrier est donc un Towaho, un ennemi. Yakati-ro, le meurtrier-chanteur, parle alors de soi-même tout en répétant les paroles de sa victime, lesquelles sont une citation de ce que

${ }^{7}$ Le mot est une probable contraction de : towãñã oho, "ennemi monstrueux *. Towã̃nã, qui n'existe pas en araweté comme lexème autonome, pourrait renvoyer à la forme tupi-guarani usuelle pour * ennemi * (tovajar, towayat, etc.). 
lui serait en train de dire : le tueur 'répete' donc ses propres mots. On se trouve devant ce qu'on pourrait appeler un processus de réverbération : un ennemi mort cite sa victime araweté (vers $n^{\circ} 1$ ) et ensuite cite son propre tueur (vers $n^{\circ} 5-8$ ), tout cela à travers les paroles de ce dernier, qui 'cite' globalement ce que sa victime à lui est en train de proférer. Tous ceux qui sont cités sont, tout compte fait, des Araweté : le mort Moiwito, le tueur Yakati-ro, mais cela toujours du point de vue d'un tiers, la victime ennemie. Il faudra se demander : qui parle dans cette chanson? Qui est le mort, qui est l'ennemi ?

On peut examiner un autre exemple, un peu plus 'lyrique' : il s'agit d'un chant appris à Kañin-widin-no par l'esprit d'un homme de la tribu asurini qu'il a blessé au début des années 70 :

1. " "Le faucon tata se réjouit"

2. s'écria la cotinga (perchée) sur l'archet ;

3. "il est content d'être sur la branche de yocin",

4. ainsi l'a entendu ma femme ;

5. "La petite lame de bambou se détourne,

6. elle se détourne de nous ;

7. elle s'éloigne de notre chemin"

8. ainsi causait ma femme. "

Ici l'ennemi qui a échappé vivant aux flèches de Kañin-widin-no se réjouit de son sort. Il répète ce que sa femme a dit ou entendu. La première partie du chant évoque le petit faucon tata sautillant joyeux sur une branche de l'arbre yocin; c'est une cotinga, perchée sur l'arc du chanteur, qui le raconte à la femme de l'ennemi. La seconde partie célèbre la mauvaise visée du chanteur, dont la fleche (" petite lame de bambou », allusion ironique puisque la pointe des fleches araweté est une pièce d'environ $60 \mathrm{~cm}$ de long) se détourne de l'ennemi et de sa femme. Ici aussi l'ennemi cite sa femme.

Lors de la transcription de ce chant, l'anthropologue demanda à ses amis araweté : mais quand l'Asurini fut atteint par la fleche il se trouvait seul dans la forêt ; qui est-ce donc cette femme dont il parle? Ils lui ont répondu qu'il s'agissait de Kañin-widin-hi, la femme du guerrier araweté ; " ma femme " désignait ainsi l'épouse du chanteur, mais celui qui était en train de dire "ma femme " était l'esprit de l'ennemi. Le chant est énoncé de son point de vue : les flèches se 
détournent de lui. Mais le régime d'énonciation fait que le chanteur, tout en se référant à sa femme comme «ma femme ", ne fait que citer des mots de l'ennemi. Nous avons déjà vu les précautions que le tueur doit prendre en ce qui concerne le sexe, tout de suite apres le meurtre : sa femme devient effectivement la femme potentielle de son ennemi.

\section{Le destin du meurtrier}

Cette réverbération entre le tueur et sa victime qui donne origine à la situation paradoxale de la danse de guerre, moment de la plus grande 'effervescence collective' chez les Araweté, où la communauté masculine se rassemble autour du tueur pour, en s'identifiant a lui, répéter les mots énoncés par un autre, est un processus qui a son prix. La fusion entre l'ennemi et son meurtrier entraine ce dernier dans un devenir-autre : l'esprit de sa victime ne le lâche jamais. Dès qu'il tue son ennemi, le meurtrier doit être dépouillé de ses armes car l'esprit du défunt, pris du désir de vengeance, lui inspire une fureur aveugle, capable de lui faire tuer ses propres parents. Un guerrier demeure exposé à ce danger assez longtemps après son exploit : souvent il est pris de crises de folie qui doivent être apaisées par ses amies rituelles (les épouses des apihi-pihă, voir ci-dessus). Parfois il doit s'enfuir dans la forêt : l'ennemi " lui jette du duvet sur la tête " et lui trouble les sens. «Quand il s'empare du tueur, l'esprit de l'ennemi le transforme en un ennemi à nous " disent les Araweté. L'ennemi ne peut se venger directement sur son tueur car il en fait partie; il essaie alors de tuer les concitoyens de son double. Vraisemblablement ce n'est que des années plus tard qu'il retrouve le calme et laisse la paix à son homicide.

Les moropl'na araweté sont vus comme des hommes dangereux, capables de recourir à la force quand on les agace, ce qui les distingue des marin-in me' $e$, des gens du commun ou " inoffensifs " (tous les non tueurs), qui exhibent d'habitude un remarquable contrôle de soi. Le 
statut de meurtrier, quoiqu'il n'accorde pas de privilèges rituels ${ }^{8}$, est sans aucun doute très honorable, et les moropi'na sont l'objet d'admiration et d'une certaine crainte ; les six hommes qui portent aujourd'hui ce titre dans la société araweté se trouvent parmi les rares individus qui ne subissent jamais de railleries de la part de leurs concitoyens, auxquelles n'échappe personne, pas même les chamans renommés. L'effondrement démographique dû à la « pacification " en 1976 a fait que les Araweté ont perdu en peu de temps huit moropl'nă, ce qu'ils ne cessent de regretter. Autrefois, disent-ils, «tous les hommes étaient des meurtriers, sans exception ". Déclaration certainement exagérée, mais qui exprime un état des choses vu comme idéal.

La différence métaphysique de la personne du meurtrier ne se révèle pleinement que dans l'au-delà. Etre dévoré après sa mort est le sort de tout individu, mâle ou femelle, chaman ou homme du commun. Seul le statut de moropi'nă met son titulaire à l'abri du cannibalisme divin. L'esprit du tueur monte aux cieux fusionné à l'esprit de sa victime. Là ils se transforment en Iraparadr, un genre d'entité spirituelle que les Mat craignent et respectent. Un Iraparadr, l'âme d'un tueur araweté accrue de son supplément ennemi, n'est pas dévoré par les dieux ; il passe directement par le bain d'immortalité, devenant un être incorruptible sans être soumis à l'épreuve de la mort cannibale. Il est d'ailleurs concevable que le meurtrier échappe à l'épreuve de la mort : on dit de plusieurs guerriers de l'antiquité qu'ils ne sont pas morts, qu'ils sont montés aux cieux en chair et en os. Idée exprimée parfois de façon dogmatique : «Un meurtrier ne meurt jamais ».

Nous avons vu que, lorsqu'il tue son ennemi, le meurtrier « meurt * et du coup ressuscite dans l'ici-bas. Dorénavant on peut dire qu'il est immortel ; voilà pourquoi il n'est pas dévoré à son arrivée aux cieux. Il est en lui-même un cannibale (son ventre est rempli du sang de son ennemi) ; et il est déjà un ennemi, une fusion complexe des attributs bide et awin. Bref, il est un Mar. Le meurtrier est un dieu anticipe : il incarne la figure de l'Ennemi tout en étant l'Araweté idéal.

${ }^{8}$ Sa seule marque visible est la frange de cheveux irrégulière : l'esprit de l'ennemi est censé faire dégarnir le front de son homicide. 
La consommation cannibale des morts aux cieux est la condition de leur transformation en êtres immortels doués d'un corps glorieux et incorruptible. Mais comme le meurtrier est un autre car il est un ennemi, il a déjà eu son apothéose. Dans l'antiquité exemplaire, les tueurs montaient aux cieux dans leurs corps ; aujourd'hui le corps enseveli d'un meurtrier pourrit, comme tout le monde peut s'en rendre compte, mais on dit que son cadavre ne produit pas le fantôme terrestre méchant et répulsif qui émane de tout cadavre. Ou alors on dit que les moropl'na morts ne produisent qu'un fantôme * inoffensif ", au contraire des fantômes des morts du commun (ceux qui de leur vivant étaient, à l'inverse des meurtriers, inoffensifs).

En tant que transformation finale de la condition de meurtrier, le concept de Iraparadi se montre essentiellement comme une perspective. Si les dieux cannibales sont à la fois l'équivalent céleste des Araweté et une figuration de l'Ennemi, s'ils nous regardent avec des yeux d'ennemi et si nous les voyons comme ennemis, la perspective de l'Iraparadimontre les Araweté se regardant activement comme ennemis. Cette capacité de se voir du point de vue de l'autre qui est peut-être l'angle idéal de la vision de soi-même nous semble la clé de l'anthropophagie tupi-guarani. Après tout, il est possible que le cannibale soit toujours l'autre (Clastres \& Lizot, 1978 : 126) ; mais qu'est-ce un Iraparadl sinon l'autre des autres, un ennemi des dieux qui de ce fait même devient, comme ceux-ci, un maître du point de vue céleste ?

L'idéal selon lequel autrefois tous les hommes étaient des tueurs révèle implicitement une situation où seules les femmes seraient dévorées par les dieux ${ }^{9}$, c'est-à-dire il suggère que le statut de « nourriture des dieux " (Mat demido, épithète qui décrit la condition humaine) est féminin et que, par conséquent, la condition de vivant humain est femelle. Le mort type est donc une femme, comme l'immortel par excellence est un meurtrier. Cette immortalité idéale ne laisse pas d'être paradoxale puisqu'un meurtrier est un homme mort, quelqu'un qui ne

${ }^{9}$ Les âmes des enfants non plus ne sont pas dévorées par les Maï : en arrivant au cieux elles sont frottées avec le jus d'un fruit qui leur régénère la peau (le bain d'immortalité où sont plongées les dépouilles des morts adultes est connu aussi par le nom de *bain de mue de la peau *) et les transforme sur le coup en jeunes adultes. 
réalise pleinement ses potentialités que dans ce double rapport avec la mort. Le meurtrier meurt quand il tue son ennemi, s'identifie à lui, et ne tire profit effectivement de ces deux morts que quand il meurt vraiment : confronté aux dieux, il n'est pas traité en ennemi, car il est un ennemi et donc d'emblée un Mar.

A l'époque où nous avons vécu chez les Araweté, le statut de tueur était moins important et illustre que celui de chaman. N'importe quel homme adulte capable de se rappeler les chants pouvait suppleer le meurtrier dans le rôle du chantre pendant les fêtes de caouïn. En revanche, l'exercice quotidien du chamanisme incombait toujours à des hommes qui disposaient du pouvoir intransmissible de manifester la voix des dieux.

Ce renversement dans la hiérarchie des deux façons d'être masculines peut être attribué à la paix qui règne aujourd'hui chez les Arawete ; mais je pense qu'il a plutôt un fondement structurel. Le chaman peut être un 'mort anticipé' en racontant ses voyages aux cieux, il rappelle toujours que les dieux parlent de lui comme de leur « future proie "; mais il joue un rôle vital et social : il est un être-pour-legroupe. Le meurtrier, en revanche, s'il est un " dieu anticipé ", manifeste un rôle mortel et individuel : il est un être-pour-soi. Le chaman est le vivant par excellence, celui qui représente les vivants aux cieux, et les morts sur la terre. Médiateur toujours distinct de ce qu'il communique (les chants chamaniques ne mélangent jamais la voix des dieux et des morts à celle du chaman), il met en communication ce qui est séparé. Son efficacité dépend du fait d'être en vie et de faire participer les morts au commerce des vivants. Quant au meurtrier, il ne représente personne mais il est l'incarnation de l'ennemi, avec qui il se confond ; il est le lieu d'une métamorphose complexe qui ne bénéficie qu'à lui. Il est clair que l'idéal d'une société formée de guerriers est présent dans la culture araweté et a dû être doublement vital dans son histoire, toujours marquée par la guerre. Mais, sous l'angle de l'eschatologie personnelle, le meurtrier est quelqu'un qui est déjà de l'autre côté, devenu ennemi et, donc, divinité. C'est pourquoi, si les chamans sont aux morts ce que les tueurs sont aux dieux, les chamans sont aux vivants ce que les tueurs sont aux morts. La société serait impossible 
sans le chamanisme ; la masculinité serait impensable sans la figure du tueur.

Les Mal sont a la fois chamans et tueurs, vie et mort. Ils sont l'archétype du chaman car ils détiennent la science magique de la résurrection ; de même, ils sont l'archétype du meurtrier puisqu'ils s'assimilent les morts à travers la dévoration, tout comme l'homicide, qui est lui aussi une fusion ambivalente de bide et de awin, transforme l'ennemi tout en se transformant en lui.

\section{Perspectives amazoniennes}

A l'exception de la nature relativement élaborée du jeu ventriloque qu'on retrouve dans les chansons d'ennemi, les matériaux araweté sur les relations meurtrier-victime étonnent par leur simplicité, si on les compare à ce qu'on connaît des enjeux symboliques et imaginaires de la violence guerrière dans d'autres sociétés d'Amérique tropicale. Cette apparente simplicité peut être attribuée, indépendamment des lacunes de l'ethnographie, au « style " général de cette société qui, peu disposée à de grands raffinements rituels, a plutôt joué le rôle de victime que celui d'agresseur vis-à-vis des différentes tribus ennemies auxquelles elle a dû s'affronter pendant les dernières décennies : la valeur attribuée a la condition de tueur chez les Araweté ne signifie point qu'ils soient particulièrement belliqueux ou particulierement performants comme guerriers.

Il est même probable que la prédation ontologique de l'extérieur comme condition de la reproduction sociale, thème que l'on rencontre dans de nombreuses sociétés d'Amazonie (Viveiros de Castro, 1993a), joue un rôle moins important chez les Araweté que, par exemple, chez les Tupinamba, les Jivaro ou les Mundurucu. Ce qui s'explique peut-être par le mouvement de translation observable dans la cosmologie araweté : les contenus symboliques qui, dans d'autres sociétés amérindiennes, sont véhiculés par le complexe guerrier se trouvent, chez les Araweté, en grande partie déplacés vers la relation entre les dieux et les hommes ; l'espace et les fonctions de l'extériorité furent appropriés par les Mal. Une comparaison avec les Tupi du XVI $\mathrm{Xl}^{\mathrm{e}}$ siecle renforce 
cette interprétation (Viveiros de Castro, 1992a, 1993b) : la sociologie cannibale des Tupinamba, déployée à travers un système rituel sophistiqué de capture, de captivité, d'exécution et de consommation des ennemis-beaux-frères, devient chez les Araweté une théologie et une eschatologie qui, bien qu'encore marquées par le langage de l'affinité et du cannibalisme, présentent un rendement institutionnel nettement inférieur à leur richesse idéologique.

Ceci dit, cette simplicité nous permettra d'aborder directement certains éléments essentiels du couple meurtrier-victime dans les sociétés amazoniennes. En guise de conclusion, voilà quelques comparaisons et réflexions d'ensemble.

\section{Les rites d'homicide}

Les matériaux araweté manifestent la prégnance d'un complexe symbolique assez répandu en Amazonie (et certainement ailleurs) qui comprend les éléments suivants : (a) l'état de 'danger mystique' où se trouve le tueur après son exploit, demandant des précautions qui cherchent à empêcher des phénomènes létaux de 'ricochet', provoqués directement ou indirectement par la victime ; $(b)$ le commerce spirituel entre le tueur et sa victime ; (c) le parallélisme entre les processus qui se déroulent dans le corps du meurtrier et dans celui de sa victime ; (d) un ensemble de rites d'homicide conçus comme transformation du sang de l'ennemi, à dominante soit anabolique (digestion, sublimation en d'autres substances vitales) soit catabolique (vomissement, saignée) ; (e) interdit de contact entre le meurtrier et la dépouille de l'ennemi : en particulier là où le cannibalisme est effectivement pratiqué, le tueur ne peut jamais manger sa victime, laquelle subit différents processus d'appropriation par la communauté du tueur ; $(f)$ abstinence sexuelle du tueur pendant sa réclusion, en général combinée à d'autres restrictions alimentaires et de conduite, isomorphes en certains cas à la couvade et, en d'autres, présentant un parallélisme explicite avec les restrictions liées à la menstruation ; ( $g$ ) accroissement du " capital ontologique " du meurtrier à la fin de sa réclusion, marqué par une 
relation de contrôle ou d'assimilation de certains attributs métonymiques de la victime (esprit, noms, chants, trophees).

De cet ensemble, nous ne prendrons ici que quelques aspects. Tout d'abord, les choses se passent comme si l'accroissement de la puissance spirituelle et sociale du meurtrier dépendait d'une période préalable où il se trouve assujetti aux influences corporelles de sa victime, ou aux pouvoirs spirituels de celle-ci en tant qu'inhérents à certaines substances vitales. Les processus d'élaboration du sang ennemi doivent donc être envisagés dans une double perspective : ils manifestent non seulement une transformation de l'ennemi effectuée dans le tueur, mais aussi une transformation $d u$ tueur menée par sa victime. L'idée selon laquelle le tueur « meurt * après son exploit suggère une aliénation du meurtrier, sa « capture » par l'image de la victime ${ }^{10}$. N'oublions pas que la réclusion post-homicide, surtout quand elle tient lieu de rite de passage permettant l'accès à des statuts prestigieux (adulte apte à se marier, leader rituel, chef de guerre), se sert abondamment des symboles classiques de mort et de résurrection : liminarité, silence, nudité, abandon du nom... Dans ce sens la mort de la victime est en effet la mort du tueur, et la renaissance de l'un est la renaissance de l'autre.

Ensuite, les règles qui empêchent un commerce intense entre le tueur et sa proie humaine sont évidentes dans les sociétés qui pratiquaient le cannibalisme rituel; en revanche, dans les sociétés où la réduction des victimes en trophées immangeables (têtes réduites, dents, scalps) jouait un rôle plus considérable, il semble qu'il n'y ait pas eu d'interdits concernant la manipulation de ces morceaux de corps par le tueur $^{11}$. La prohibition de consommation cannibale de la victime par son tueur rappelle immédiatement l'interdit amazonien, si répandu, qui empêche le chasseur de manger sa proie, injonction souvent interprétée

${ }^{10}$ Comparer, par exemple, le pourrissement du meurtrier araweté, sa digestion du sang ennemi et son obsession par l'esprit de la victime avec le cannibalisme du tueur yanomami, qui vomit la graisse et les cheveux de la victime (signe qu'il a mangé son âme) et qui devient possédé par le * principe vital * de celle-ci (Lizot, $1976: 13$ \& 228; Albert, 1985 : 360-ss).

11 Pour le premier cas, voir Viveiros de Castro (1992a:282-ss) sur les Tupinamba et Vilaça (1992 : 101-15) sur les Pakaa-Nova ; pour le second cas, voir Taylor (1993a) sur les Jivaro, Menget (1993a) sur les Mundurucu et Sterpin (1993) sur les Nivacle. 
comme une exigence de réciprocité banissant l' « auto-consommation ", analogue à l'inceste. Cela semble s'appliquer aux proies humaines; d'ailleurs, dans les sociétés pratiquant la chasse aux têtes, les trophées devaient être socialisés, autrement dit ils devaient bénéficier à toute la communauté du tueur (en leur apportant l'abondance, la fécondité, une protection contre les ennemis, du prestige). Ceci dit, il faut s'interroger sur les conditions de possibilité de cette équivalence, du point de vue de la logique du don, entre proies humaines et animales.

L'évidente continuité technique et symbolique entre la chasse et la guerre en Amazonie a été assez longtemps refoulée chez les anthropologues, peut-être parce que la reconnaître impliquerait l'attribution aux cultures de la région d'une attitude d' " animalisation » de l'ennemi ; et de là à expliquer la guerre indigène en termes davantage éthologiques qu'ethnologiques il n'y aurait qu'un pas. Mais de telles conséquences inacceptables ne se produisent que si l'on adopte un point de vue radicalement étranger aux conceptions indigènes. L'animalisation de l'ennemi qu'on décele dans l'équivalence entre chasse et guerre dépend d'une première et plus fondamentale humanisation de l'animal. Ẹn bref : en Amazonie indigène, les relations entre société et nature ne sont pas conçues comme des relations naturelles, mais comme des relations sociales $^{12}$. Guerre et chasse sont, littéralement, un même combat : combat qui oppose des êtres sociaux, c'est-à-dire des sujets. Sous cet aspect, il n'y a effectivement pas de discontinuité entre la prédation cynégétique et la prédation guerrière ; l'aliénation rituelle du meurtrier ne differe guère, de par sa nature, de cette identitication dangereuse entre le chasseur et sa proie leur imposant une disjonction dans l'ordre de la consommation, c'est-à-dire au moment de l'« objectivation » de la proie. Cet aspect éminemment subjectif et agonistique du rapport entre prédateur et proie (humaine ou animale) fonde la réversibilité qui l'accompagne toujours et qui ne fait qu'exprimer la mutuelle détermination entre tueur et victime.

Enfin, les précautions rituelles imposées au tueur, dans la mesure où elles se justifient par une conception du sang en tant qu'inducteur

12 Pour cette idée, voir Viveiros de Castro, $1992 b$ et surtout Descola, 1992. 
ou signe d'un changement de statut métaphysique, mettent en scène les femmes. On a beaucoup écrit sur le rôle fondamental de la communauté féminine dans les rituels guerriers et dans le cannibalisme ; à çe sujet, la pertinence des observations de Lévi-Strauss (1984) a été largement vérifiée dans le cas de l'Amazonie. Par ailleurs, l'équivalence symbolique ou l'enchaînement causal entre, d'une part, les conditions masculines associées à l'imposition de la mort et, d'autre part, les conditions féminines liées à la production de la vie (homicide et menstruation, réclusion rituelle et gestation, guerre et mariage..) est l'un de ces themes qu'on retrouve un peu partout, en Amazonie comme en Polynésie, en Nouvelle-Guinée ou dans la Grèce ancienne. Il convient de remarquer que, dans certains cas amazoniens tout au moins, de telles analogies suggèrent un potentiel de féminisation du meurtrier, sa « fécondation * ou «possession» par la victime, situation qui doit être rituellement transmuée en un pouvoir spécifiquement masculin de création. Cela suggère encore une autre série d'analogies entre la transformation rituelle des ennemis (conçus comme des identités capturées de l'extérieur) et l'engendrement d'enfants (la production de nouvelles identités à l'intérieur du groupe).

\section{Intériorisation et extériorisation}

Nous avons pu détecter une progression dans le cas araweté : dans une situation initiale caractérisée par la plus grande distance sociale et la plus petite distance physique, la mort violente de l'ennemi déclenche une « catastrophe fusionnelle » donnant lieu à une identification instable entre les deux pôles de la relation, qui aboutit à l'englobement métonymique de la victime par son tueur. Ce processus se retrouve, avec d'innombrables variations, chez d'autres sociétés amazoniennes. Dans le cas araweté, la victime se transforme en appendice spirituel du meurtrier, devenant son bouclier ou son embleme dans le milieu cannibale du ciel ; dans d'autres cultures (dont les Nivacle sont un bon exemple ; Sterpin, $1993: 49-50$ ), elle est conçue comme un « traître ", un élément étranger qui est passé au camp ennemi, celui du tueur, où elle joue le rôle d'animal domestique, c'est-à-dire de subjectivité 
extérieure sauvage qui a été « apprivoisée » et détournée de sa nature première $^{13}$. Dans d'autres cas, l'humanité de la victime est entièrement recyclée dans de nouveaux composants du groupe : ainsi le meurtrier pakaa-nova transforme le sang ennemi en sperme et pourra engendrer un fils qui sera l'incarnation de l'esprit de sa victime, ce qui parachève la transmutation de l'extériorité cannibale et prédatoire en consubstantialité, soins paternels et commensalité (Vilaça, 1992 : 101-15) ; les rites jivaro de célébration de la tête-trophée, à leur tour, aboutissent à la transformation de celle-ci en un fils générique (plus exactement un fotus) des femmes du groupe (Taylor, 1993a) ; de même, les têtestrophées mundurucu étaient vues métaphoriquement comme enfants d'une « mère " masculine (le meurtrier). Ľes Mundurucu illustrent, d'ailleurs, un autre thème commun à la région (Menget, 1993a) : la tête de l'ennemi mort était préparée et décorée de façon à devenir l'image ethnique d'un homme du groupe ; cela fait penser aux Tupi du XVI siècle, chez qui le prisonnier de guerre était laborieusement épilé, peint et paré à l'image de ses futurs bourreaux.

Nous nous trouvons ainsi devant un processus général d'assimilation de la victime à la personne du meurtrier, ou, de façon plus générale, au groupe de ce dernier. Ce processus semble être fonction, en certains cas, de la reconnaissance préalable de la victime comme ressemblant sous quelques aspects à ses agresseurs ; ainsi, seulement les étrangers « a bonne distance " sont considérés comme des proies légitimes - les Jivaro, par exemple, ne prenaient des têtes que dans les sous-groupes de la même ethnie (Taylor 1985). Mais l'assimilation

${ }^{13}$ Les scalps des Nivacle révélaient à leurs maîtres les mouvements de l'ennemi, c'est-à-dire, des groupes d'où provenaient les victimes. Les crânes-trophées des Shipaya avaient la même fonction de délation (Nimuendaju, 1981 : 23-24), ainsi que l'esprit des Kayapo tués par les Tapirapé, qui devenaient familiers aux chamans, les prévenant des attaques des Kayapo vivants (Wagley, 1977: 184-85). Chez les Araweté, cette inversion du comportement de la victime apparaît dans le cas du jaguar. L'esprit d'un jaguar mort reste à côté de son tueur, devenant une sorte de chien de chasse: il dort au-dessous de son hamac et lui montre en rêve des endroits où le gibier abonde. Le chant traditionnel qui célèbre la mort du jaguar obéit à la même réverbération que les chansorıs d'ennemi: le nom de ce chant est * future victime du jaguar * et il met en scène un jaguar qui parle des humains qu'il va manger dans une double inversion, logique et temporelle, du point de vue. 
peut aussi produire cette ressemblance a posteriori, comme c'est le cas chez les Tupinamba et les Mundurucu, où l'ennemi était " nationalisé " avant d'être soumis aux procédés rituels. Cette combinaison de différences et ressemblances également nécessaires souvent se cristalise dans l'identification des ennemis comme étant des affins : les " beaux-frères ennemis " tupinamba (H. Clastres, 1972) ne sont que l'exemple le plus célèbre d'une configuration amérindienne très généralisée, où la tension caractéristique de l'affinité, relation qui a la ressemblance pour base et la différence pour principe, est utilisée dans le but de penser la catégorie de l'ennemi et vice-versa, c'est-à-dire où les valeurs de l'extériorité prédatoire forment le " sous-texte * de l'alliance matrimoniale (Viveiros de Castro, 1993a).

Or, toutes ces idées présupposent l'humanité intégrale de la victime, sa condition d'altérité pleinement subjective ; cela revient à dire que la relation entre tueur et victime ne peut engendrer toutes ces identifications mystiques et fusions rituelles que si elle est d'emblée conçue et vécue comme relation sociale. Mais, d'un autre côté, la définition ou production rituelle de l'ennemi comme sujet, le processus de subjectivation de l'Autre nécessaire à son assimilation par le Même, contient en soi, presque comme sa condition, le moment inverse : l'objectivation du meurtrier, son altération par la victime, son identification à l'ennemi comme ennemi. Nous l'avons déjà trouvé dans le processus de réverbération mis en oeuvre dans les chants de guerre araweté : le meurtrier s'appréhende comme sujet à partir du moment où il se voit soi-même à travers le regard de sa victime, ou plutôt, où il prononce sa singularité à lui par la voix de celle-ci. Il est possible de le confirmer ailleurs ; la poétique araweté de l'altérité trouve son écho dans l'esthétique wayana de l'extériorité : quand ils partent en guerre, les hommes wayana doivent se faire scarifier avec des motifs représentant des jaguars et des oiseaux de proie, figures qui manifestent la nature prédatoire propre aux ennemis (van Velthem, 1995 : 254-55). Nous avions déjà remarqué la même chose à propos de la décoration corporelle des $\mathrm{Mal}$.

Tout semble converger, en somme, vers une " étonnante indistinction entre l'agresseur et la victime, au profit d'une sorte d'essence de la guerre ", comme le remarque P. Menget $(1993 b: 29)$ à propos des Txicao. L'intériorisation de l'Autre est inséparable de l'extériorisation 
du Soi ; la domestication de celui-là va de pair avec l'ensauvagement de celui-ci.

On peut se demander aussi ce qui est au fond assimile quand on assimile l'ennemi. Les ethnographes d'Amazonie font mention de " ressources " symboliques très variées et qui sont loin de s'exclure mutuellement dans chaque configuration culturelle : noms, chants, substances spirituelles, énergies vitales, identités, visages, principes d'individuation... Il n'est pas question de reproduire ici des arguments déjà exposés à propos du cannibalisme tupinamba (Viveiros de Castro, $1992 a$ ) ; nous ne présenterons que les conclusions de ce travail, qui d'ailleurs s'accordent bien avec ce qu'on a pu voir en analysant les chansons d'ennemi des Araweté. Plutôt que des substances ou principes plus ou moins réifiés que chaque société (ou chaque ethnographe) choisit comme substrat et objet des processus d'assimilation de l'ennemi, nous pensons que ce qui est en jeu est l'incorporation de quelque chose d'éminemment incorporel : le statut même d'ennemi. Ce qu'on assimile de la victime, ce sont les signes de son altérité, et ce qu'on vise, c'est cette altérité comme point de vue ou perspective sur le Soi, c'est-à-dire une relation. Mais si ce que l'on dévore, de façon réelle ou imaginaire, de la personne de l'ennemi est cette perspective sur le Soi du groupe agresseur, on peut dire que le socius se constitue dans l'interface avec son extérieur ou, en d'autres termes, qu'il se pose comme déterminé essentiellement par l'extériorité. En choisissant comme principe de mouvement l'incorporation d'attributs venant de l'ennemi, la société amérindienne est amenée à se définir selon ces mêmes attributs, ce qu'on peut voir à l'œuvre aux grands moments rituels de la vie de ces sociétés, où la place d'honneur est toujours réservée à la figure bifrons du meurtrier et de sa victime, qui se reflètent et se réverbèrent à l'infini.

\section{Prédation ontologique et fusion rituelle}

S'il est bien vrai que « le point de vue crée l'objet ", comme le disait Saussure, il n'est pas moins vrai que le point de vue crée le sujet, une fois que la position de sujet se définit précisément par la faculté d'avoir un point de vue. Dans ce sens, le substantivisme de l'assimila- 
tion des proprietés de la victime amérindienne ne correspond pas tellement à une économie ontologique imaginaire mais davantage à une “ épistémologie ontologisée " (Taylor, 1993b : 444-45) où les transformations produites par l'agression guerrière s'effectuent avant tout sur des relations et des statuts cognitivement déterminés, c'est-à-dire sur des perspectives. En avançant que la dynamique identitaire du couple meurtrier-victime dans les sociétés amazoniennes est un processus d'assomption du point de vue de l'ennemi, nous cherchons à comprendre ce qui rend à la fois profondément familières et radicalement étranges les représentations amérindiennes de la guerre. Loin d'impliquer le traitement de l'ennemi comme "chose " (système physique, corps anonyme, automate animal), le devenir du couple meurtrier-victime suppose un engagement entre sujets, engagement qui ne doit pas être appréhendé sous le mode hégélien d'une lutte de consciences, mais comme une dialectique où tous sont des Maîtres qui échangent des points de vue et alternent des moments de subjectivation et d'objectivation : objectivation du tueur par la subjectivité de la victime quand celle-ci le possède, contrôle et «tue"; subjectivation du tueur par l'objectivité de la victime, quand celle-ci est décantée en des chants, noms, trophées et autres synecdoques qui expriment la nouvelle condition ontologique du meurtrier. On peut appliquer à la violence guerrière amazonienne ce que Simon Harrison, dans un ouvrage récent, disait de son analogue mélanésien :

« Aggression is conceived as very much a communicative act directed against the subjectivity of others, and making war required the reduction of the enemy, not to the status of a non-person or thing but, quite the opposite, to an extreme state of subjectivity (1993: 121). "

Cela signifie que l'inimitié, dans ce type de société, "is conceptualised not as a mere objective absence of a social relationship but as a definite social relationship like any other» (id. : 128). Ceci évoque naturellement un passage assez connu de Lévi-Strauss (1967 : 552-53) :

« [L]es observateurs ont été souvent frappés par l'impossibilité, pour les indigenes, de concevoir une relation neutre, ou plus exactement une absence de relation [...] l'absence de relation familiale ne définit 
pas rien, elle définit l'hostilité [...] il n'est pas davantage possible de se tenir en deçà, ou au delà, du monde des relations..."

Four les indigènes, donc, aucune différence n'est indifférente, toute différence est immédiatement relation douée de positivité ; l' « hostilité " n'est pas rien, mais, au contraire, elle est une relation socialement déterminée. On peut pourtant avancer un peu plus : le schéma général de toute différence, comme le montre amplement l'ethnologie amazonienne, est la prédation cannibale dont l'affinité est une codification spécifique, bien que privilégiée. Malgré le risque de tomber dans un excès allégorique, on peut affirmer que, dans ces cosmologies, la proposition attributive générique est une proposition cannibale ; le paradigme de la relation prédicative entre sujet et objet est la prédation et l'incorporation : entre affins, entre les sexes, entre vivants et morts, entre humains et animaux, entre humains et esprits, et, naturellement, entre ennemis. La copule de toute proposition synthétique a priori, dans cet univers articulé par une " logique des qualités sensibles", est en effet une copulation charnelle ou carnivore. Sujet et objet s'interconstituent par la prédation incorporante, dont la réciprocité caractéristique indique, d'ailleurs, l'inexistence de positions absolues (du sujet comme substance, du prédicat comme accident $)^{14}$.

Il faut le répéter : ces relations de prédation sont d'emblée des relations sociales. La réciprocité (réelle et symbolique) inhérente à la prédation cannibale suggère qu'il s'agit ici d'une prédation subjectivante, interne au " monde des relations " dont parlait Lévi-Strauss. Elle n'a rien à voir avec la production objectivante moderne qui suppose justement une relation neutre (impossible dans les cosmologies amérindiennes) où un Sujet humain actif regarde un Autre inerte et naturalisé.

14 Voir Overing, 1986 : 90-95, pour une analyse du cannibalisme comme 'vérité' profonde de toute relation sociale chez les Piaroa. Parmi les études qui mettent en relief l'importanee de la prédation ontologique en Amazonie, voir les travaux d'Albert (1985; 1988) sur les Yanomami et ceux de Vilaça (1992) sur les Pakaa-Nova. Albert a analysé avec profusion de détails le symbolisme de la prédation cannibale comme langage de base des relations politiques (c'est-à-dire supra-locales) yanomami, identifiant encore une intéressante configuration de différents degrés et modalités de prédation selon les niveaux de la classification socio-politique, différenciation qui fait appel à plusieurs techniques agressives et touche à différentes composantes personnelles. 
La prédation généralisée amérindienne est un personnage du monde du don et de la "lutte des hommes " (Lefort, 1978), et non pas du monde du travail et de l'assujettissement aux choses.

Les relations entre le meurtrier et sa victime appartiennent sans doute au monde du don, mais elles y occupent une position-limite : si la synthèse du don relie des sujets qui demeurent objectivement séparés, la mise à mort violente et sa symbolique cannibale peuvent, à l'inverse, être perçues comme productrices d'une synthèse où toute distance est supprimée : la relation est crée à partir de l'abolition de l'un de ses termes, qui se voit introjecté par l'autre ; la dépendance réciproque qui relie et constitue les sujets de l'échange se mue ici en fusion, ou, si l'on veut, elle objective des termes qui deviennent subjectivement indissolubles. Comme l'observe Harrison à propos des Mélanésiens qu'il a étudiés :

" Just as a gift embodies the identity of its donor, so in Lowland warfare the killer acquires through homicide an aspect of his victim's identity. The killing is represented as either creating or expressing a social relationship, or else as the collapse of a social relation by the merging of two social alters into one (1993:130). "

C'est un processus qui pourrait être nommé - si l'on fait appel au concept de schismogenèse proposé par Bateson (1958) et récemment réinterprété par Houseman \& Severi (1994) comme fondement de la logique rituelle du naven iatmul - " antischismogenèse rituelle " car il semble viser moins à une différenciation progressive des points de vue des participants du rite qu'à un mouvement de rapprochement fusionnel et d'introjection des différences. Au lieu de se montrer comme un terme déployé en valeurs opposées, le " monopôle rituel " qu'est le tueur se forme par repliement, par une espèce de condensation d'attributs où la double négation (« je suis l'ennemi de mon ennemi ») ne restitue pas l'identité initiale, mais la déplace d'un cran (« je suis l'ennemi »).

L'agression guerrière amérindienne se présente alors comme (pour employer encore une fois les termes de Harrison) un processus de transformation rituelle du Soi. Ce qui nous met devant le versant obscur de la pensée sauvage : elle nous remet au sacrifice, à l'« animisme " et à la métonymie, et non pas au totémisme, au « symbolisme » et à la 
métaphore $^{15}$. Si les figures du totémisme articulent des séries qui restent distinctes des relations qui les unissent, à leur tour les figures sacrificielles comme celle du devenir meurtrier-victime visent à la transformation d'une série dans l'autre, opération * absolueou extrême » (Lévi-Strauss, 1962 : 298) marquée par la continuité, l'ambiguïté et l'irréversibilité. Mais si le meurtrier et sa victime semblent être plutôt du côté de chez Lévy-Bruhl que du côté de chez Lévi-Strauss, il ne faut pas oublier que, tout comme Méséglise et Guermantes, il y a plus d'un chemin qui relie ces directions, lesquelles de toute façon, comme l'a montré leur réunion finale, n'étaient pas aussi éloignées que le croyait le point de vue, fatalement subjectif, du Narrateur.

Eduardo Viveiros de Castro Musée National, Rio de Janeiro (texte traduit du portugais par Estela dos Santos Abreu)

\section{Références bibliographiques}

Albert, Bruce

1985 Temps du sang, temps des cendres : représentation de la maladie, système rituel et espace politique chez les Yanomami du sud-est (Amazonie brésilienne). Thèse de doctorat, Université de Paris X (Nanterre).

Bateson, Gregory

1958 Naven : A survey of the Problems Suggested by a Composite Picture of the Culture of a New Guinea Tribe drawn from three Points of View, Stanford, Stanford University Press, (1 1 ère éd. 1936).

15 Ce versant n'a pas encore été exploité systématiquement dans l'ethnologie amazonienne, trop marquée par le biais 'totémiciste' et classificatoire : voir Viveiros de Castro, 1992a:11, 28, 273-278 et Descola, $1992: 113-15$. 
Clastres, Hélène

1972 "Les Beaux-frères ennemis : à propos du cannibalisme tupinamba. * Nouvelle Revue de Psychanalyse, 6, Destins du cannibalisme, 71-82.

Clastres, Hélène \& Lizot, Jacques

1978 * La Part du feu : rites et discours de la mort chez les Yanomami. * Libre, 3, 135-149.

Descola, Philippe

1992 "Societies of Nature and the Nature of Society * in Conceptualizing Society, A. Kuper ed., London, Routledge, 107-126.

Harrison, Simon

1993 The Mask of War: Violence, Ritual and the Self in Melanesia. Manchester, Manchester University Press.

Houseman, Michael \& Carlo, Severi

1994 Naven ou le donner à voir : essai d'interprétation de l'action rituelle, Paris, Maison des Sciences de l'Homme / CNRS.

Lefort, Claude

1978 * L'échange et la lutte des hommes * in Les formes de l'histoire : essais d'anthropologie politique, Paris, Gallimard, (1 ${ }^{\mathrm{e} r e}$ éd. 1951), 15-29.

Lévi-Strauss, Claude

1967 Les Structures élémentaires de la parenté, Paris, Mouton (1 $1^{\text {rre }}$ éd. 1949)

1962 La Pensée sauvage, Paris, Plon.

1984 Paroles données, Paris, Plon.

Lizot, Jacques

1976 Le Cercle des feux : faits et dits des indiens Yanomami, Paris, Seuil.

Menget, Patrick

$1993 a$ * Notas sobre as cabeças mundurucu * in Amazônia : etnologia e história indigena, E. Viveiros de Castro \& M. Carneiro da Cunha (sous dir.), São Paulo, Núcleo de História Indígena e do Indigenismo / USP, 311-321.

$1993 b$ * Le Propre du nom : remarques sur l'onomastique txicão *, Journal de la Société des Américanistes, LXXIX, 21-31.

Nimuendaju, Curt

1981 "Fragmentos de religião e tradição dos índios Shipaya *, Religião \& Sociedade, 7, (1 ${ }^{\text {ère }}$ éd. 1949), 347. 
Overing, Joanna

1986 * Images of Cannibalism, Death and Domination in a 'Non-violent' Society ", in The Anthropology of Violence, D. Riches (sous dir.), London, Basil Blackwell, 86-102.

Price, David

1987 * Nambiquara Geopolitical Organisation * Man, 22, 1, 1-24.

Sterpin, Adriana

1993 * La Chasse aux scalps chez les Nivacle du Gran Chaco *, Journal de la Société des Américanistes, LXXIX, 33-66.

Taylor, Anne Christine

1985 * L'art de la réduction *, Journal de la Société des Américanistes, LXXI, 159-173.

$1993 a$ * Les Bons ennemis et les mauvais parents : le traitement symbolique de l'alliance dans les rituels de chasse aux têtes des Jivaros de l'Equateur» In Les Complexités de l'alliance, IV. Économie, politique et fondements symboliques de l'alliance, E. Copet \& F. Héritier-Augé Eds, Paris, Archives Contemporaines.

$1993 b$ * Des Fantômes stupéfiants : langage et croyance dans la pensée achuar *, L'Homme 126-128, (2-4), 429-447.

Van Velthem, Lúcia

1995 O Belo é a fera : a estética da produção e da predação entre os Wayana, Thèse de doctorat, Université de São Paulo.

Vilaça, Aparecida

1992 Comendo como gente : formas do canibalismo wari', Rio de Janeiro, Editora da UFRJ.

Viveiros de Castro, Eduardo

1992 a From the Enemy's Point of View : Humanity and Divinity in an Amazonian Society, Chicago, The University of Chicago Press, (1 ${ }^{\text {ère }}$ éd. 1986).

$1992 b$ * Sociedades indígenas e natureza na Amazônia *, Tempo $e$ Presença, 14, 261, 25-26.

$1993 a$ * Alguns aspectos da afinidade no dravidianato amazônico * in Amazônia : etnologia e história indigena, E. Viveiros de Castro \& M. Cameiro da Cunha eds, São Paulo, Núcleo de História Indígena e do Indigenismo / USP, 150-210.

$1993 b$ * Le Marbre et le myrte : de l'inconstance de l'âme sauvage *, in Mémoire de la tradition, A. Becquelin \& A. Molinié (sous dir.), Nanterre, Société d'Ethnologie, 365-431.

Wagley, Charles

1977 Welcome of Tears : The Tapirapé Indians of Central Brazil, New York, Oxford University Press. 